\title{
GROUP INVARIANT SOLUTION OF SOME TIME FRACTIONAL EVOLUTION EQUATIONS
}

\author{
Hemanta Mandal, B. Bira \\ Department of Mathematics, SRM Institute of Science and Technology \\ Chennai, India \\ bibekananda.b@ktr.srmuniv.ac.in
}

Received: 20 June 2018; Accepted: 31 January 2019

\begin{abstract}
In this paper, we consider some classes of a system of nonlinear fractional differential equations (FDEs) arising in some important physical phenomena. Using symmetry group of transformations, the given systems of fractional partial differential equations (FPDEs) are reduced to systems of fractional ordinary differential equations (FODEs). Further, using the group invariant condition, we solve the reduced systems of FODEs and exact solutions of the given equations are constructed. Finally, the physical significance of the solutions are investigated graphically based on the exact solutions in order to highlight the importance of the study.
\end{abstract}

MSC 2010: 35R11, 76M60

Keywords: fractional coupled Burger's equation, fractional DSSH equations, Riemann Liouville's fractional derivative, Erdélyi-Kober operator, Symmetry analysis, exact solution

\section{Introduction}

In recent years, the study of FDEs arising in nonlinear phenomena has drawn much attention of many researchers, both from mathematical and physical points of view. Many nonlinear coupled evolution equations with fractional order have been established in order to describe a variety of non-classic phenomena such as fluid mechanics, viscoelasticity, signal processing, systems identification, control theory, finance and fractional dynamics [1-3]. Due to the wide applications in the field of natural and social sciences, this subject emerges as a hot topic of research. The most important advantage of using FDEs in these and other applications is their nonlocal property. Building fractional mathematical models for specific phenomena and developing numerical or analytical solutions for these fractional mathematical models are crucial issues in mathematics, physics, and engineering. The development of the subject is contemporary to the classical calculus and was started with the letter of L'Hopital's to Liebniz asking, for the $n$-th order derivative of the linear function $f(x)=x$ is $\frac{D^{n} x}{D x^{n}}$, what would the result be if $n=1 / 2$ ? Later, Liouville, Riemann, 
Grunwald, Caputo, Letnikov, Jumarie etc extended it to an arbitrary order derivative.

In order to better understand these phenomena as well as further apply them in practical life, it is important to seek their more exact solutions. However, given FDEs, there exists no well-defined method to analyze and study them systematically as well as dealing with exact explicit solutions. Many methods have been successfully used by many engineers and scientific researchers to handle such problems. For example, the solution of PDEs of fractional order, using the adomian decomposition method is found in [4]. Whereas, the use of finite difference methods for the time FDEs, the author refers to [5,6]. The authors in [7], used the generalized differential transform method and obtained the numerical solutions of the space-and time-fractional coupled Burgers equations. The fractional variational iteration method and its application is found in [8]. In [9], the sub-equation method has been used by the author to find the exact solutions of nonlinear time fractional differential equations.

On the other hand, the FPDEs can be solved analytically using the application of Lie group analysis. Lie symmetry analysis is one of the most powerful and systematic methods, which plays a very important role in finding an exact solution of such nonlinear coupled evolution equations. For the theory of Lie group analysis and its applications to differential equations we refer [10-13]. There are only few papers which are devoted to group analysis of FDEs and group properties of FDEs are much less understood. Scaling transformations of the time fractional linear wave-diffusion equation and its group invariant solutions have been described in [14]. Using group of scaling transformations, self similarity solutions to $\mathrm{KdV}$ system of equations are obtained in [15]. Lie symmetries of the fractional nonlinear anomalous diffusion equations are studied in [16]. Complete group classification and symmetry reductions of the fractional fifth-order KdV type of equations are performed in [17, 18].

The structure of the work in this article is organized as: in Section 2, we recall some definitions and properties of the integral calculus which are used in the preceding sections. In Section 3, we present the application of the Lie group analysis to derive the symmetric group of transformations under which the given systems of equations remain invariant. In Section 4, as the application of the method we take some physical examples and construct the particular exact solution for the corresponding problems exhibiting the space and time relationship. Further, the nonlinear property of the solutions with respect to fractional order derivative $\alpha$ is discussed with the help of $2 D$ and $3 D$-plots. Finally, in Section 5, we state our conclusion.

\section{Preliminaries}

In this section, we recall some definitions and properties of the fractional calculus theory which may be used in our study and for more details we refer to $[19,20]$.

\subsection{Definition}

(a) Riemann-Liouville fractional derivative: The Riemann-Liouville fractional 
differential operator of order $\alpha>0$ of $\mathrm{f}(\mathrm{t})$ is given as

$D_{t}^{\alpha} f(x)=\left\{\begin{array}{lc}\frac{1}{\Gamma(n-\alpha)} \frac{d^{n}}{d t^{n}} \int_{0}^{t}(t-\tau)^{n-\alpha-1}(f(\tau)) d \tau, & n-1<\alpha<n, \quad n \in N \\ \frac{d^{n}}{d t^{n}} f(t), & \alpha=n \in N,\end{array}\right.$

(b) Caputo fractional derivative: For $\alpha \in(n-1 ; n)$, the fractional derivative of $\mathrm{f}$ is given as

$$
{ }_{*} D_{t}^{\alpha} f(t)=\frac{1}{\Gamma(n-\alpha)} \int_{0}^{t}(t-\xi)^{n-\alpha-1}\left(f^{n}(\tau)\right) d \tau, \quad n-1<\alpha<n, \quad n \in N
$$

\subsection{Properties of Riemann-Liouville fractional derivative}

Here we recall some properties for the modified Riemann-Liouville derivative which are used in the proceeding work:

(i) $D_{t}^{\alpha} t^{\gamma}=\frac{\Gamma(\gamma+1)}{\Gamma(\gamma+1-\alpha)} t^{\gamma-\alpha}, \quad \gamma>n$.

(ii) $D_{t}^{\alpha}[f(x) g(x)]=\sum_{n=0}^{\infty}\left(\begin{array}{l}\alpha \\ k\end{array}\right) D_{t}^{x} f(t) D_{t}^{\alpha-n} g(x), \quad\left(\begin{array}{l}\alpha \\ k\end{array}\right)=\frac{\Gamma(\alpha+1)}{\Gamma(k+1) \Gamma(\alpha+1-k)}$.

(iii) $D_{a}^{\alpha} f(g(x))$

$$
=\frac{(x-a)^{-\alpha}}{\Gamma(1-\alpha)} f(g(x))+\sum_{m=1}^{k}\left(\begin{array}{l}
\alpha \\
k
\end{array}\right) \frac{k !(x-a)^{k-\alpha}}{\Gamma(k-\alpha-1)} \sum_{k=1} f^{(m)}(g(x)) \sum \prod_{r=1}^{k} \frac{1}{a_{r} !}\left({\frac{g^{(r)}(x)^{a_{r}}}{r !}}^{r !},\right.
$$

where the sum extends over all combinations of non-negative integer values of $a_{1}, a_{2}, a_{3} \ldots a_{k}$ such that $\sum_{r=1}^{k} r a_{r}=n$ and $\sum_{r=1}^{k} a_{r}=m$.

\section{Symmetry analysis of fractional PDEs}

This section deals with the brief discussion of lie symmetry analysis for nonlinear FPDEs with two independent variables and one dependent variable. We consider the 
one parameter Lie group of transformation as follows:

$$
\begin{aligned}
x^{*} & =x+\varepsilon \xi^{x}(x, t, u)+O\left(\varepsilon^{2}\right), \\
t^{*} & =t+\varepsilon \xi^{t}(x, t, u)+O\left(\varepsilon^{2}\right), \\
u^{*} & =u+\varepsilon \eta(x, t, u)+O\left(\varepsilon^{2}\right), \\
\frac{\partial^{\alpha} \bar{u}}{\partial \bar{t}^{\alpha}} & =\frac{\partial^{\alpha} u}{\partial t^{\alpha}}+\varepsilon \eta_{\alpha}^{0}(x, t, u)+O\left(\varepsilon^{2}\right), \\
\frac{\partial \bar{u}}{\partial \bar{x}} & =\frac{\partial u}{\partial x}+\varepsilon \eta^{x}(x, t, u)+O\left(\varepsilon^{2}\right), \\
\frac{\partial^{2} \bar{u}}{\partial \bar{x}^{2}} & =\frac{\partial^{2} u}{\partial x^{2}}+\varepsilon \eta^{x x}(x, t, u)+O\left(\varepsilon^{2}\right),
\end{aligned}
$$

where

$$
\begin{aligned}
\eta_{\alpha}^{0}= & \frac{\partial^{\alpha} \eta}{\partial t^{\alpha}}+\left(\eta_{u}-D_{t}\left(\xi^{t}\right)\right) \frac{\partial^{\alpha} u}{\partial t^{\alpha}}-u \frac{\partial^{\alpha} \eta_{u}}{\partial t^{\alpha}}+\mu \\
& +\sum_{n=1}^{\infty}\left[\left(\begin{array}{l}
\alpha \\
n
\end{array}\right) \frac{\partial^{n} \eta_{u}}{\partial t^{\alpha}}-\left(\begin{array}{l}
\alpha \\
n+1
\end{array}\right) D_{t}^{n+1}\left(\xi^{t}\right)\right] D_{t}^{\alpha-n}(u) \\
& -\sum_{n=1}^{\infty}\left(\begin{array}{l}
a \\
n
\end{array}\right) D_{t}^{n}\left(\xi^{x}\right) D_{t}^{\alpha-n}\left(u_{x}\right)
\end{aligned}
$$

and

$$
\begin{aligned}
\mu & =\sum_{n=2}^{\infty} \sum_{m=2}^{n} \sum_{k=2}^{m} \sum_{r=0}^{k-1}\left(\begin{array}{l}
a \\
n
\end{array}\right)\left(\begin{array}{l}
n \\
m
\end{array}\right)\left(\begin{array}{l}
k \\
r
\end{array}\right) \frac{1}{k !} \frac{t^{n-\alpha}}{\Gamma(n+1-\alpha)}[-u]^{r} \frac{\partial^{m}}{\partial t^{m}}\left[u^{k-r}\right] \frac{\partial^{n-m+k} \eta}{\partial t^{n-m} \partial u^{k}} \\
\eta^{x} & =D_{x}(\eta)-u_{x} D_{x}\left(\xi^{x}\right)-u_{t} D_{x}\left(\xi^{t}\right), \\
\eta^{x x} & =D_{x}\left(\eta^{x}\right)-u_{x t} D_{x}\left(\xi^{t}\right)-u_{x x} D_{x}\left(\xi^{x}\right), \\
\eta^{x x x} & =D_{x}\left(\eta^{x} x\right)-u_{x x t} D_{x}\left(\xi^{t}\right)-u_{x x x} D_{x}\left(\xi^{x}\right),
\end{aligned}
$$

Here $D_{x}$ denotes total order derivative and denoted as

$$
D_{x}=\frac{\partial}{\partial x}+u_{x} \frac{\partial}{\partial u}+u_{x x} \frac{\partial}{\partial u_{x}}+u_{x x x} \frac{\partial}{\partial u_{x x}}+\cdots
$$

The general vector field can be considered as

$$
V=\xi^{t}(x, t, u) \frac{\partial}{\partial t}+\xi^{x}(x, t, u) \frac{\partial}{\partial x}+\eta(x, t, u) \frac{\partial}{\partial u},
$$


and we take the prolongation of this vector field depending on the highest order derivative present in the given equation.

\section{Application of the method}

As the application of Section 3, we take the following examples and construct the group invariant solutions.

Example 1: The well known coupled Burger's equation with time fractional derivatives, describing flow of stock wave under a viscous fluid is considered as [21]:

$$
\begin{aligned}
& \frac{\partial^{\alpha} u}{\partial t^{\alpha}}=u_{x x}+k_{1} u u_{x}-u v_{x}-v u_{x}, \\
& \frac{\partial^{\alpha} v}{\partial t^{\alpha}}=v_{x x}+k_{1} v v_{x}-u v_{x}-v u_{x},
\end{aligned}
$$

where $u(x, t)$ and $v(x, t)$ represents the wave profiles, the variables $x$ and $t$ respectively represent the normalized space and time variables. The parameter $\alpha$ is standing for the order of the fractional time derivative satisfying $0<\alpha<1$.

Following the straightforward calculation as in Section 3, we get the infinitesimal transformations as follows

$$
\xi^{x}=\alpha C_{5} x+C_{1}, \quad \xi^{t}=2 C_{5} t+C_{3}, \quad \eta^{u}=-\alpha C_{5} u, \quad \eta^{v}=-\alpha C_{5} v,
$$

where $C_{1}, C_{3}, C_{5}$ are arbitrary constants. The infinitesimal generators associated with (2), can be written as

$$
X_{1}=\frac{\partial}{\partial x}, \quad X_{3}=\alpha x \frac{\partial}{\partial x}+2 t \frac{\partial}{\partial t}-\alpha u \frac{\partial}{\partial u}-\alpha v \frac{\partial}{\partial v}, \quad X_{5}=\frac{\partial}{\partial t} .
$$

For the symmetry reduction we consider the generator $X_{3}$ and the associated characteristic can be written as below:

$$
\frac{d x}{\alpha x}=\frac{d t}{2 t}=\frac{d u}{-\alpha u}=\frac{d v}{-\alpha v},
$$

From $\frac{d t}{2 t}=\frac{d u}{-\alpha u}$, we get $u=t^{-\frac{\alpha}{2}} U$.

Where $U$ is a function of $\xi$. Continuing similar calculations, we get the following similarity variables

$$
u=t^{-\frac{\alpha}{2}} U(\xi), \quad v=t^{-\frac{\alpha}{2}} V(\xi), \quad \xi=x t^{-\frac{\alpha}{2}} .
$$

The use of the above similarity variables in the sense of Riemann-Liouville derivatives, the governing system of FPDEs (1), can be reduced to a nonlinear system of FODEs, through the following theorem. 
Theorem: The similarity variables in (3) reduces (1) to the following nonlinear system of FODEs:

$$
\begin{aligned}
& {\left[\left(1-\frac{3 \alpha}{2}-\frac{\alpha}{2} \xi \frac{d}{d \xi}\right)\left(F_{\beta}^{-\frac{\alpha}{2}, \frac{\alpha}{2}} U\right)(\xi)\right]=U_{\xi \xi}+k_{1} U U_{\xi}-U V_{\xi}-V U_{\xi}} \\
& {\left[\left(1-\frac{3 \alpha}{2}-\frac{\alpha}{2} \xi \frac{d}{d \xi}\right)\left(F_{\beta}^{-\frac{\alpha}{2}, \frac{\alpha}{2}} V\right)(\xi)\right]=V_{\xi \xi}+k_{1} V V_{\xi}-U V_{\xi}-V U_{\xi}}
\end{aligned}
$$

Proof: For $0<\beta<1$,

$$
\begin{aligned}
& \frac{\partial^{\beta} u}{\partial t^{\beta}}=\frac{1}{\Gamma(1-\beta)} \frac{\partial}{\partial t} \int_{0}^{t}(t-s)^{-\beta}(f(s)) d s \\
= & \frac{1}{\Gamma(1-\beta)} \frac{\partial}{\partial t} \int_{0}^{t}(t-s)^{-\beta} s^{-\frac{\alpha}{2}} U\left(x s^{-\frac{\alpha}{2}}\right) d s
\end{aligned}
$$

and considering $c=\frac{\alpha}{2}, d=\frac{\alpha}{2}$, one can derive the Riemann-Liouville fractional derivatives for the system of equations (1) as

$$
\frac{\partial^{\beta} u}{\partial t^{\beta}}=\frac{1}{\Gamma(1-\beta)} \frac{\partial}{\partial t} \int_{0}^{t}(t-s)^{-\beta} s^{-d} U\left(x s^{-c}\right) d s .
$$

Putting $\tau=\frac{s}{t}$ and $d \tau=\frac{1}{t} d s$, we get

$$
\begin{aligned}
\frac{\partial^{\beta} u}{\partial t^{\beta}} & =\frac{1}{\Gamma(1-\beta)} \frac{\partial}{\partial t}\left[\int_{0}^{1}(1-\tau)^{-\beta} t^{1-d-\beta} \tau^{-d} U\left(\xi \tau^{-c}\right) d \tau\right] \\
& =\frac{\partial}{\partial t}\left[t^{1-d-\beta}\left(F_{\beta}^{-d, c} U\right)(\xi)\right]
\end{aligned}
$$

where

$$
\left(F_{\beta}^{-d, c} U\right)(\xi)=\frac{1}{\Gamma(1-\beta)} \int_{0}^{1}(1-\tau)^{-\beta} \tau^{-d} U\left(\xi \tau^{-c}\right) d \tau .
$$

is the Erdelyi-Kober fractional integral operator.

Using chain rule,

$$
\frac{\partial}{\partial t}=\frac{d}{d \xi} \frac{\partial \xi}{\partial t}=-\frac{\alpha}{2} \xi t^{-1} \frac{d}{d \xi}=-c \xi t^{-1} \frac{d}{d \xi},
$$

with which equation (6) can be rewritten as

$$
\frac{\partial^{\beta} u}{\partial t^{\beta}}=t^{-d-\beta}\left[\left(1-d-\beta-c \xi \frac{d}{d \xi}\right)\left(F_{\beta}^{-d, c} U\right)(\xi)\right]
$$


In the present work, as we take $\alpha=\beta, c=\frac{\alpha}{2}$ and $d=\frac{\alpha}{2}$, hence we obtain

$$
\frac{\partial^{\alpha} u}{\partial t^{\alpha}}=t^{\frac{-3 \alpha}{2}}\left[\left(1-\frac{3 \alpha}{2}-\frac{\alpha}{2} \xi \frac{d}{d \xi}\right)\left(F_{\beta}^{-\frac{\alpha}{2}, \frac{\alpha}{2}} U\right)(\xi)\right] \text {. }
$$

Similarly,

$$
\frac{\partial^{\alpha} v}{\partial t^{\alpha}}=t^{\frac{-3 \alpha}{2}}\left[\left(1-\frac{3 \alpha}{2}-\frac{\alpha}{2} \xi \frac{d}{d \xi}\right)\left(F_{\beta}^{-\frac{\alpha}{2}, \frac{\alpha}{2}} V\right)(\xi)\right]
$$

Further, the reduced system of FODEs is obtained as

$$
\begin{aligned}
& {\left[\left(1-\frac{3 \alpha}{2}-\frac{\alpha}{2} \xi \frac{d}{d \xi}\right)\left(F_{\alpha}^{-\frac{\alpha}{2}, \frac{\alpha}{2}} U\right)(\xi)\right]=U_{\xi \xi}+k_{1} U U_{\xi}-U V_{\xi}-V U_{\xi}} \\
& {\left[\left(1-\frac{3 \alpha}{2}-\frac{\alpha}{2} \xi \frac{d}{d \xi}\right)\left(F_{\alpha}^{-\frac{\alpha}{2}, \frac{\alpha}{2}} V\right)(\xi)\right]=V_{\xi \xi}+k_{1} V V_{\xi}-U V_{\xi}-V U_{\xi}}
\end{aligned}
$$

Exact solution: Here we construct the exact group invariant solution of the timefractional coupled Burger's equation (1) as outlined in [22]. For that, let us first introduce a function as below

$$
U(\xi)=A \xi^{a}, \quad V(\xi)=B \xi^{b}, \quad \xi=x t^{-c},
$$

where $A, B, a$ and $b$ are arbitrary real constants are to be determined explicitly. Applying the formula

$$
F_{\beta}^{a, b}\left(\xi^{k}\right)=\frac{1}{1-\beta} \int_{0}^{1}(1-\tau)^{-\beta} \tau^{a} \xi^{k} \tau^{-b k} d \tau=\frac{\Gamma(1+a-b k)}{\Gamma(2+a-\beta-b k)} \xi^{k}
$$

Substituting (9) in (7), we get

$$
\begin{aligned}
& \frac{\Gamma\left(1-\frac{\alpha}{2}-\frac{\alpha}{2} a\right)}{\Gamma\left(1-\frac{3 \alpha}{2}-\frac{\alpha}{2} a\right)} A \xi^{a}=A a(a-1) \xi^{a-2}+A^{2} a \xi^{2 a-1}-A B a \xi^{a+b-1}+A B a \xi^{a+b-1} \\
& \frac{\Gamma\left(1-\frac{\alpha}{2}-\frac{\alpha}{2} b\right)}{\Gamma\left(1-\frac{3 \alpha}{2}-\frac{\alpha}{2} b\right)} B \xi^{b}=B b(b-1) \xi^{b-2}+B^{2} b \xi^{2 b-1}-A B a \xi^{a+b-1}-A B b \xi^{a+b-1}
\end{aligned}
$$

The exact group invariant solution of a system of equations (10) will exist if this system of equations remains invariant with respect to the variable $\xi$. It can be clearly noticed that the system of equations (10) will remain invariant with respect to $\xi$ if $a=b=1$. Hence the value of the arbitrary constants $A, B$ can be obtained as,

$$
\begin{aligned}
& A=-\frac{\Gamma(1-\alpha)}{\Gamma(1-2 \alpha)}, \\
& B=-\frac{\Gamma(1-\alpha)}{\Gamma(1-2 \alpha)},
\end{aligned}
$$


which in turn produces the solution of original system of FPDEs as follows

$$
\begin{aligned}
& u=-\frac{\Gamma(1-\alpha)}{\Gamma(1-2 \alpha)}\left(x t^{-\frac{\alpha}{2}}\right), \\
& v=-\frac{\Gamma(1-\alpha)}{\Gamma(1-2 \alpha)}\left(x t^{-\frac{\alpha}{2}}\right),
\end{aligned}
$$

where the solution is defined.
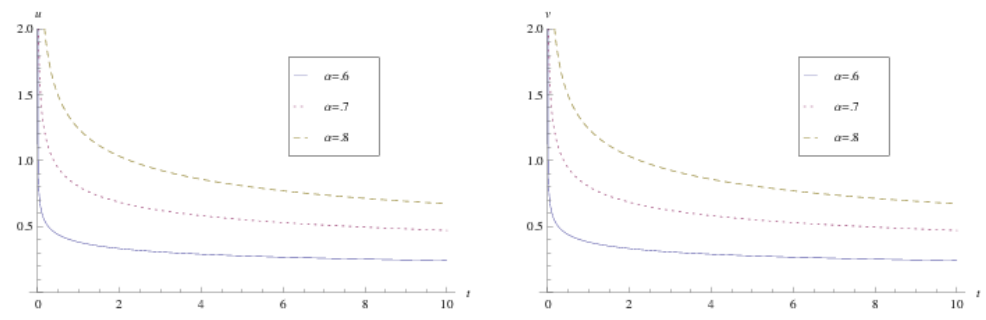

Fig. 1. Nature of the solution $u(x, t)$ and $v(x, t)$ for fixed $x$ and $\alpha=0.6, \alpha=0.7$ and $\alpha=0.8$ respectively
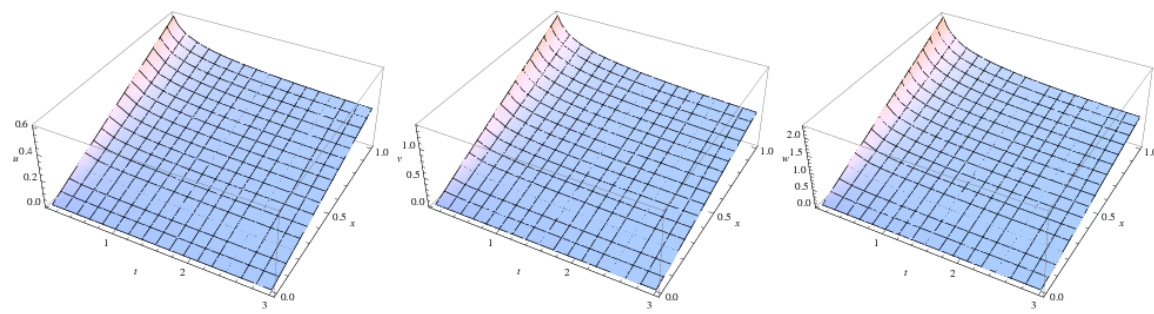

Fig. 2. 3D-plot of $u(x, t)$ for $\alpha=0.6, \alpha=0.7$ and $\alpha=0.8$

Example 2: As the next example, we consider the time-fractional coupled Drinfeld-Sokolov-Satsuma-Hirota (DSSH) as [23]:

$$
\begin{aligned}
\frac{\partial^{\alpha} p}{\partial t^{\alpha}}-\frac{1}{2} p_{x x x}+3 p p_{x}-3 q_{x} & =0, \\
\frac{\partial^{\alpha} q}{\partial t^{\alpha}}+q_{x x x}-3 p q_{x} & =0
\end{aligned}
$$

where $p(x, t)$ and $q(x, t)$ are the dependent variables and the variables $x$ and $t$ representing space and time respectively are the independent variables. The parameter $\alpha$ 
stands for the order of the fractional time derivative with $0<\alpha<1$.
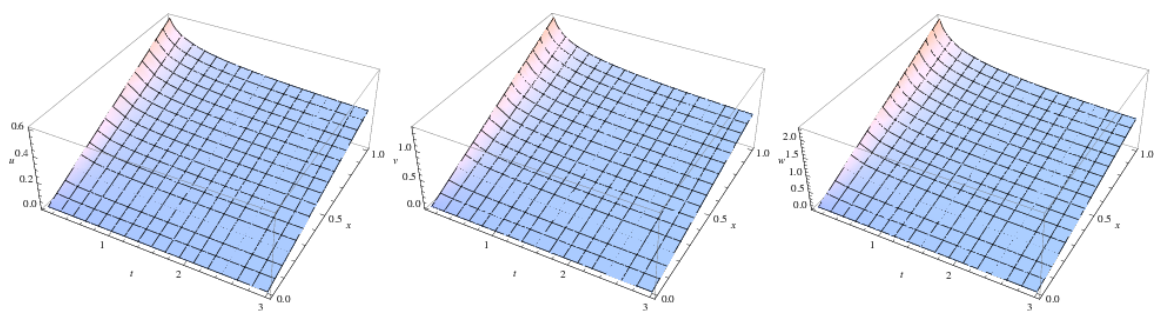

Fig. 3. 3D-plot of $v(x, t)$ for $\alpha=0.6, \alpha=0.7$ and $\alpha=0.8$

From the application of Lie group analysis and from the straight forward analysis as in Section 3, we get a symmetric group of transformations

$$
\eta^{x}=a_{3} \alpha x+a_{4}, \quad \eta^{t}=a_{1}+3 a_{3} t, \quad \phi^{p}=-2 \alpha a_{3} p, \quad \phi^{q}=-4 \alpha a_{3} q+F_{8}(x, t),
$$

where $a_{1}, a_{2}, a_{3}$ are arbitrary constants. $F_{8}(x, t)$ is function of the variable $x$ and $t$. We consider the finite dimensional Lie algebra for our computational purpose and the infinitesimal generators associated with $a_{1}, a_{2}$ and $a_{3}$ are given as follows:

$$
\begin{aligned}
& Y_{1}=\frac{\partial}{\partial t}, \quad Y_{3}=\frac{\partial}{\partial x}, \\
& Y_{2}=\alpha x \frac{\partial}{\partial x}+3 t \frac{\partial}{\partial t}-2 \alpha p \frac{\partial}{\partial p}-4 \alpha q \frac{\partial}{\partial q} .
\end{aligned}
$$

Since $F_{8}(x, t) \frac{\partial}{\partial q}$ is infinite dimensional, so we study only finite dimensional generators.

For the symmetric reduction of equation (12), we consider scaling transformation $X_{2}$ and we get the similarity variables by the method of characteristic as

$$
p=t^{-\frac{2 \alpha}{3}} P(\eta) \quad q=t^{-\frac{4 \alpha}{3}} Q(\eta) \quad \eta=x t^{-\frac{\alpha}{3}} .
$$

Using the similarity variables from (15) and repeating the above analysis, the system of FPDEs (12) can be reduced to a nonlinear system of ODEs of fractional order. As a result, we get the following

$$
\begin{aligned}
& {\left[\left(1-\frac{5 \alpha}{3}-\frac{\alpha}{3} \eta \frac{d}{d \xi}\right)\left(F_{\beta}^{-\frac{2 \alpha}{3}, \frac{\alpha}{3}} P\right)(\eta)\right]=\frac{1}{2} P_{\eta \eta \eta}-3 P P_{\eta}+3 Q_{\eta}} \\
& {\left[\left(1-\frac{7 \alpha}{3}-\frac{\alpha}{3} \eta \frac{d}{d \xi}\right)\left(F_{\beta}^{-\frac{4 \alpha}{3}, \frac{\alpha}{3}} Q\right)(\eta)\right]=-Q_{\eta \eta \eta}+3 P Q_{\eta} .}
\end{aligned}
$$


Exact solution: In order to have the the group invariant solution, we consider $P(\eta)=K_{1} \eta^{a}, Q(\eta)=K_{2} \eta^{b}, \eta=x t^{-c}, c=\frac{\alpha}{3}$. Following the similar analysis as in Example 1, we get

$$
\begin{aligned}
& \frac{\Gamma\left(1-\frac{2 \alpha}{3}-\frac{\alpha}{3} a\right)}{\Gamma\left(1-\frac{5 \alpha}{3}-\frac{\alpha}{3} a\right)} K_{1} \eta^{a}=\frac{1}{2} K_{1} a(a-1)(a-2) \eta^{a-3}-3 K_{1}^{2} a \eta^{2 a-1}+3 K_{2} b \eta^{b-1} \\
& \frac{\Gamma\left(1-\frac{4 \alpha}{3}-\frac{\alpha}{3} b\right)}{\Gamma\left(1-\frac{7 \alpha}{3}-\frac{\alpha}{3} b\right)} K_{2} \eta^{b}=-K_{2} b(b-1)(b-2) \eta^{b-3}+3 K_{1} K_{2} b \eta^{a+b-1}
\end{aligned}
$$
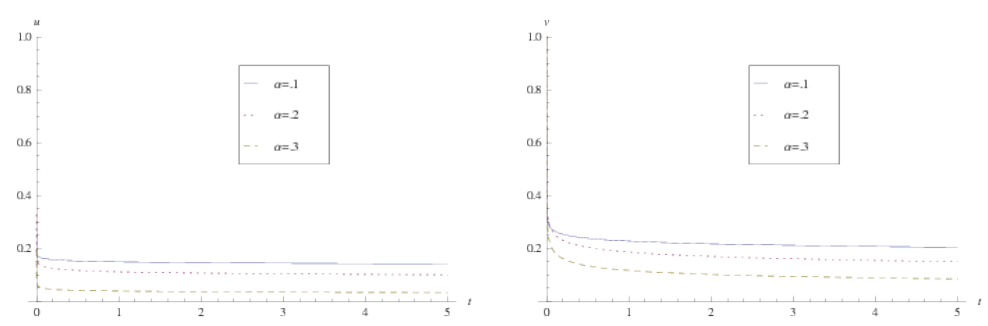

Fig. 4. Nature of the solutions $u(x, t)$ and $v(x, t)$ for fixed $x$ and $0<\alpha<1$
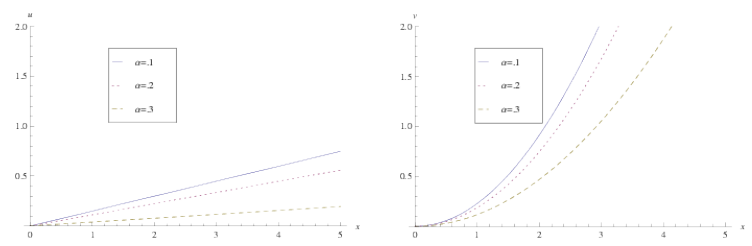

Fig. 5. Nature of the solutions $u(x, t)$ and $v(x, t)$ for fixed $t$ and $0<\alpha<1$

One can check that the reduced system of FODEs (17) will be invariant with respect to the variable $\eta$ for $a=1$, and $b=2$ whose intern yields the values of $K_{1}$ and $K_{2}$ as

$$
\begin{aligned}
& K_{1}=\frac{1}{6} \frac{\Gamma(1-2 \alpha)}{\Gamma(1-3 \alpha)} \\
& K_{2}=\frac{1}{6}\left[\frac{\Gamma(1-\alpha)}{\Gamma(1-2 \alpha)}+\frac{1}{2} \frac{\Gamma(1-2 \alpha)}{\Gamma(1-3 \alpha)}\right] .
\end{aligned}
$$

Hence the solution of original system of FPDEs (12) is given as

$$
\begin{aligned}
& u=\frac{1}{6} \frac{\Gamma(1-2 \alpha)}{\Gamma(1-3 \alpha)}\left(x t^{-\frac{\alpha}{3}}\right) \\
& v=\frac{1}{6}\left[\frac{\Gamma(1-\alpha)}{\Gamma(1-2 \alpha)}+\frac{1}{2} \frac{\Gamma(1-2 \alpha)}{\Gamma(1-3 \alpha)}\right]\left(x t^{-\frac{\alpha}{3}}\right)^{2} .
\end{aligned}
$$



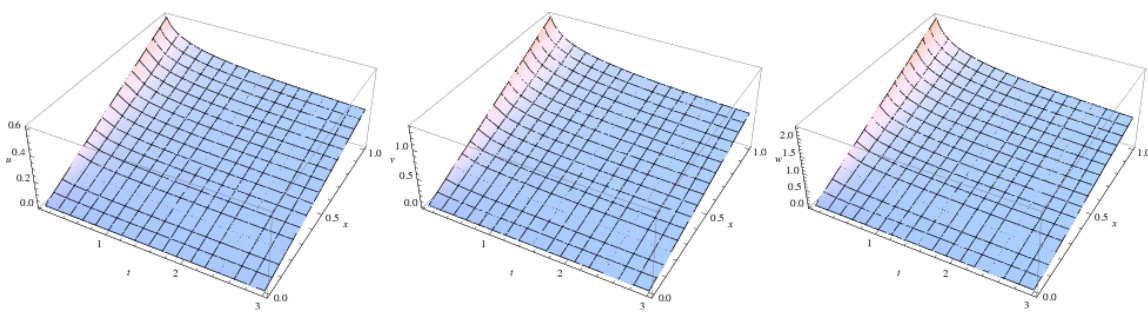

Fig. 6. $3 D$-plot of $u(x, t)$ for $\alpha=0.6, \alpha=0.625$ and $\alpha=0.65$
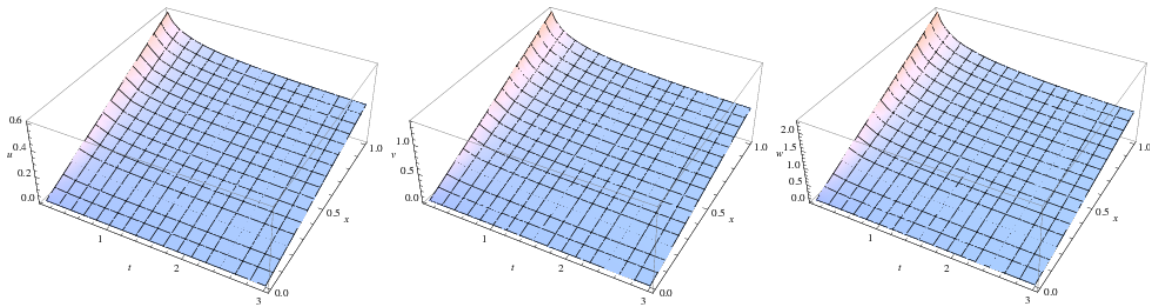

Fig. 7. $3 D$-plot of $v(x, t)$ for $\alpha=0.55, \alpha=0.575$ and $\alpha=0.6$

\section{Conclusions}

In our present study, some nonlinear time-fractional evolution equations which occur in different areas of mathematical physics are considered. From the application of the Lie group analysis we obtained a particular exact group invariant solutions for the given FPDEs. Further, the effect of the fractional order $\alpha$ on the behavior of the solutions is studied graphically. From Figures 1-7 it is observed that a change in noninteger order derivative value $\alpha$ affects the solution behavior of the solution in a fundamental way. Therefore we can conclude that the non-integer order derivative can be used to modify the shape of the wave without changing the non-linearity and the dissipative effect in the medium.

\section{Acknowledgement}

Research support from National Board for Higher Mathematics, Department of Atomic Energy, Government of India (Ref. No. NBHM/R.P.75/2015/Fresh/165) is gratefully acknowledged by the authors. 


\section{References}

[1] Kilbas, A.A., Srivastava, H.M., \& Trujillo, J.J. (2006). Theory and Applications of Fractional Differential Equations. Amsterdam: Elsevier Science B.V.

[2] Hilfer, R. (2000). Applications of Fractional Calculus in Physics. Singapore: World Scientific.

[3] Xu, X.Y., \& Tan, W.C. (2006). Intermediate processes and critical phenomena: theory, methods and progress of fractional operators and their applications to modern mechanics. Science in China Series $G, 49,257-272$.

[4] El-Sayed, A.M.A., \& Gaber, M. (2006). The Adomian decomposition method for solving partial differential equations of fractal order in finite domains. Phys Lett A, 359, 175-182.

[5] Wang, H., \& Du, N., (2014). Fast alternating-direction finite difference methods for threedimensional space-fractional diffusion equations. Journal of Computational Physics, 258, 305-318.

[6] Zhang, Y.N., Sun, Z.Z., \& Liao, H.L. (2014). Finite difference methods for the time fractional diffusion equation on non-uniform meshes. Journal of Computational Physics, 265, 195-210.

[7] Liu, J., \& Hou, G. (2011). Numerical solutions of the space-and time-fractional coupled Burgers equations by generalized differential transform method. Applied Mathematics and Computation, 217, 7001-7008.

[8] Wu, G.C., Shi, Y.G., \& Wu, K.T. (2011). Adomian decomposition method and non-analytical solutions of fractional differential equations. Romanian Journal of Physics, 56, 873-880.

[9] Bekir, A., Askoy, E., \& Cevikel, A.C. (2015). Exact solutions of nonlinear time fractional differential equations by sub-equation method. Mathematical Methods in the Applied Sciences, 38, 2779-2784.

[10] Olver, P.J. (1986). Applications of Lie Groups to Differential Equations. New York: Springer.

[11] Bluman, G.W., \& Anco, S.C. (2002). Symmetries and Integration Methods for Differantial Equations. New York: Springer-Verlag.

[12] Nayak, S., \& Chakraverty, S. (2018). Interval Finite Element Method with MATLAB. Academic Press.

[13] Chakraverty, S., \& Nayak, S. (2017). Neutron Diffusion: Concepts and Uncertainty Analysis for Engineers and Scientists. Florida: CRC Press.

[14] Buckwar, E., \& Luchko, Y. (1998). Invariance of a partial differential equation of fractional order under the Lie group of scaling transformations. Journal of Mathematical Analysis and Applications, 227, 81-97.

[15] Hu, J., Ye, Y., Shen, S., \& Zhang, J. (2014). Lie symmetry analysis of the time fractional KdVtype equation. Applied Mathematics and Computation, 233, 439-444.

[16] Gazizov, R.K., Kasatkin, A.A., \& Lukashchuk, S.Y. (2007). Symmetry properties of fractional diffusion equations. Physica Scripta, 136, 014016.

[17] Liu, H.Z. (2013). Complete group classifications and symmetry reductions of the fractional fifthorder KdV types of equations. Studies in Applied Mathematics, 131, 317-330.

[18] Yaşar, E., \& Giresunlu, İ.B. (2015). Lie symmetry reductions, exact solutions and conservation laws of the third order variant Boussinesq system. Applicable Analysis, 128, 252-255.

[19] Podlubny, I. (1999). Fractional Differential Equations. San Diego: Academic Press.

[20] Oldham, K.B., \& Spanier, J. (2006). The Fractional Calculus. New York: Dover Publications.

[21] Ali, K.K., Raslan, K.R., \& EL-Danaf, T.S. (2015). Non-polynomial spline method for solving coupled Burgers' equations. Computational Methods for Differential Equations, 3, 218-230.

[22] Costa, F.S., Marão, J.A., Soares, J.C., \& Oliveira, E.C. (2015). Similarity solution to fractional nonlinear space-time diffusion-wave equation. Journal of Mathematical Physics, 56, 033507.

[23] Geng, X., \& Li, R. (2015). Darboux transformation of the Drinfeld-Sokolov-Satsuma-Hirota system and exact solutions. Annals of Physics, 361, 215-225. 